\title{
Azygos Vein
}

National Cancer Institute

\section{Source}

National Cancer Institute. Azygos Vein. NCI Thesaurus. Code C53029.

A blood vessel which returns blood to the heart from the posterior walls of the thorax and abdomen. 